\title{
Chiral Recognition of Dansyl Derivatives with an Amino Acid-Based Molecular Micelle: A Molecular Dynamics Investigation
}

\author{
Mauro Garcia' ${ }^{1}$ Nathan Black ${ }^{1}$, Eugene Billiot ${ }^{1 *}$, Fereshteh Billiot ${ }^{1}$, Kevin F. Morris ${ }^{2}$, Yayin Fang ${ }^{3}$ \\ ${ }^{1}$ Department of Physical and Environmental Sciences, Texas A\&M University-Corpus Christi, \\ Corpus Christi, TX, USA \\ ${ }^{2}$ Department of Chemistry, Carthage College, Kenosha, WI, USA \\ ${ }^{3}$ Department of Biochemistry and Molecular Biology, Howard University, Washington, DC, USA \\ Email: *eugene.billiot@tamucc.edu
}

How to cite this paper: Garcia, M., Black, N., Billiot, E., Billiot, F., Morris, K.F. and Fang, Y.Y. (2021) Chiral Recognition of Dansyl Derivatives with an Amino AcidBased Molecular Micelle: A Molecular Dynamics Investigation. Open Journal of Physical Chemistry, 11, 64-86.

https://doi.org/10.4236/ojpc.2021.112004

Received: February 17, 2021

Accepted: May 23, 2021

Published: May 26, 2021

Copyright (c) 2021 by author(s) and Scientific Research Publishing Inc. This work is licensed under the Creative Commons Attribution International License (CC BY 4.0).

http://creativecommons.org/licenses/by/4.0/

\begin{abstract}
In this study, the chiral separation mechanisms of Dansyl amino acids, including Dansyl-Leucine (Dans-Leu), Dansyl-Norleucine (Dans-Nor), Dansyl-Tryptophan (Dans-Trp) and Dansyl-Phenylalanine (Dans-Phe) binding to poly-sodium $N$-undecanoyl-(L)-Leucylvalinate, poly (SULV), were investigated using molecular dynamics simulations. Micellar electrokinetic chromatography (MEKC) has previously shown that when separating the enantiomers of these aforementioned Dansyl amino acids, the L-enantiomers bind stronger to poly (SULV) than the D-enantiomers. This study aims to investigate the molecular interactions that govern chiral recognition in these systems using computational methods. This study reveals that the computationally-calculated binding free energy values for Dansyl enantiomers binding to poly (SULV) are in agreement with the enantiomeric order produced in experimental MEKC studies. The L-enantiomers of Dans-Leu, Dans-Nor, Dans-Trp, and Dans-Phe binding to their preferred binding pockets in poly (SULV) yielded binding free energy values of $-21.8938,-22.1763,-21.3329$ and $-13.3349 \mathrm{~kJ} \cdot \mathrm{mol}^{-1}$, respectively. The D-enantiomers of Dans-Leu, Dans-Nor, Dans-Trp, and Dans-Phe binding to their preferred binding pockets in poly (SULV) yielded binding free energy values of $-14.5811,-15.9457,-13.6408$, and $-12.0959 \mathrm{~kJ} \cdot \mathrm{mol}^{-1}$, respectively. Furthermore, hydrogen bonding analyses were used to investigate and elucidate the molecular interactions that govern chiral recognition in these molecular systems.
\end{abstract}

\section{Keywords}

Amino Acid Based Molecular Micelles, Molecular Modeling, Computational 
Chemistry, Chiral Recognition

\section{Introduction}

More than half of all pharmaceutical drugs currently in use are chiral, and the synthesis of these drugs often yields a racemic mixture, containing both enantiomers of the compound [1]. While the physical and chemical properties of the enantiomers are similar, they often produce very different pharmacological effects [1] [2] [3]. In many cases, one enantiomer may produce the desired medicinal effect, whereas the other may cause adverse health effects. For example, (+)-Ethambutol is primarily used to treat Tuberculosis, whereas its enantiomer, (-)-Ethambutol, causes blindness [4]. Due to the enantiomers of chiral drugs often producing vastly different effects, the United States Food and Drug Administration has mandated that each enantiomer of a chiral drug be tested for enantiomeric purity prior to being marketed [5]. Due to the enantiomers sharing nearly identical physical properties such as boiling point, density, mass and solubility, this often makes it difficult to separate enantiomers based on those aforementioned properties [6]. However, the enantiomers differ in stereoconfiguration, which allows them to interact differently with chiral separation mediums, thus allowing for enantioseparation [5].

Some commonly used chiral separation techniques are high-performance liquid chromatography (HPLC) and capillary electrophoresis (CE), which utilize chiral stationary and pseudostationary phases, respectively [7] [8]. In using chiral chromatographic techniques, if the two enantiomers of a chiral compound, the R- and S-enantiomer, have different binding affinities to the chiral separation medium, then enantiomeric separations can occur. While both aforementioned techniques are able to effectively separate enantiomers on the basis of their binding affinity to the chiral separation medium, CE has several advantages over HPLC. A much smaller sample size is required for CE as compared to HPLC and often, CE yields a higher number of theoretical plates, ultimately producing better enantiomeric resolution in a shorter period of time [9] [10]. In HPLC, the chiral recognition medium is covalently linked to a type of solid support material such as silicon beads [11]. Therefore, the user would need to purchase individual columns containing the desired stationary chiral recognition medium. Consequently, if the user wanted to switch out the chiral separation medium, it would entail switching out the column(s). However, in CE, the chiral recognition medium can be exchanged quite easily with another chiral medium since it is part of the mobile phase, in which the chiral medium acts as the pseudostationary phase.

Chiral selectors such as cyclodextrins, polysaccharides, crown ethers and chiral micelles have proven to be effective chiral selectors in CE [7] [12] [13]. This research will focus on the latter, chiral micelles, more specifically, a class of chir- 
al micelles known as amino acid-based micelles. When micelles are used as the pseudostationary phase in CE, the technique is commonly known as Micellar Electrokinetic Chromatography (MEKC) [8] [10] [14] [15].

Amino acid-based micelles are composed of surfactant units, each containing a hydrocarbon chain; one end of the chain contains a terminal alkane, and the opposite side of the chain is connected to an amino acid head group. Amino acid-based surfactants have advantages over other chiral selectors in that amino acids are ubiquitous therefore deeming it cheaper than other options; these surfactants are also biodegradable and environmentally-friendly [16]. These surfactants have been extensively studied using single amino acid and dipeptide head groups, in which the dipeptide micellar systems have shown significant advantages in achieving enhanced enantiomeric resolution in MEKC compared to single amino acid based micellar systems [10]. This is due in part to the increased number of chiral centers on the head group, thus allowing for enhanced chiral selectivity. In addition, previous research reveals that the dipeptide amino acid-based micelles act as better chiral selectors in their polymerized forms [15] [17]. The polymerized surfactants are formed via free radical polymerization when the surfactant solution is subjected to gamma radiation at concentrations significantly above their critical micelle concentration (CMC), usually $5-10$ times the CMC. This gamma radiation causes the terminal alkenes to undergo free radical polymerization to form covalent bonds with neighboring surfactant units (see Figure 1(a)). These polymerized micelles are commonly referred to as amino acid-based molecular micelles (AABMMs) [17]. AABMMs have significant advantages over the traditional, non-polymerized micelle, including: AABMMs do not have a critical micelle concentration (CMC), reduced joule heating and most notably enhancing the enantiomeric resolution [15].

This manuscript focuses on one particular AABMM, poly-sodium $N$-undecanoyl(L)-Leucylvalinate, or poly (SULV). Previous literature reports that when studying a wide-range of chiral compounds, enantiomeric separation was achieved for

(a)<smiles>C=CCCCCCCCCC(=O)NC(CC(C)C)C(=O)NC(C(=O)OCc1ccccc1)C(C)C</smiles>

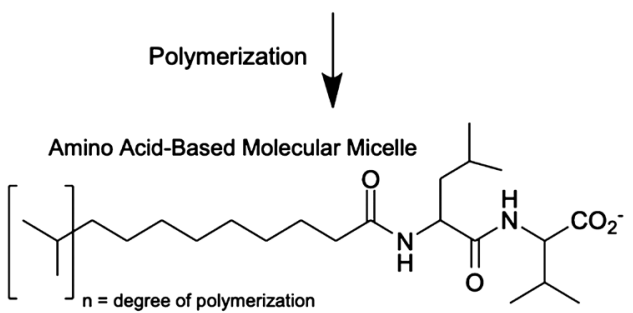

(b)<smiles>CN(C)c1cccc2ccccc12</smiles><smiles>CC(C)CC(NS(=O)(=O)O)C(=O)O</smiles>

(d)
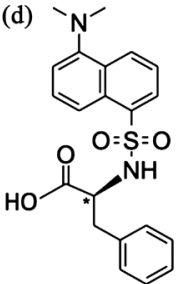

(c)

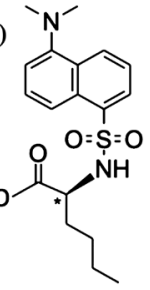

(e) ' $\mathrm{N}$ '

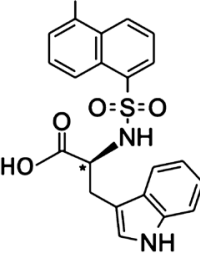

Figure 1. Chemical structures of (a) Poly-(sodium Undecyl-(L)-Leucine-Valine), (b) Dansyl Leucine, (c) Dansyl Norleucine, (d) Dansyl Phenylalanine, (e) Dansyl Tryptophan. The four Dansyl amino acids (b) - (e) contain an asymmetric center denoted by an asterisk. 
more than $75 \%$ of the compounds when using poly (SULV) as the pseudostationary phase in MEKC [14]. Chiral recognition with poly (SULV) has been examined using a variety of techniques. One of those techniques is Nuclear Overhauser Effect Spectroscopy Nuclear Magnetic Resonance (NOESY NMR), which has been used to study the primary site of interaction (s) of chiral analytes to molecular micelles [18]. Molecular dynamics (MD) simulations have also previously been used to investigate AABMMs, such as poly (SULV) to further investigate the factors that contribute to chiral recognition [5] [17]. Some of the chiral analytes previously studied with poly (SULV) include, beta blockers and binaphthyl compounds [5] [19]. This manuscript will focus on examining the binding of various Dansyl amino acids to the AABMM poly SULV.

Due to the intractable number of testing conditions and combinations that exist between: AABMMs, chiral analytes, counterions, surfactant concentration, and $\mathrm{pH}$ levels, it is important to develop a predictive model that is ultimately more cost-effective, efficient and requires less time than experimental methods. This investigation is part of a long-term project aimed at developing a Quantitative Structure-Enantioselective Retentions Relationship (QSERR) model [20] [21]. More specifically, the QSERR model will be developed to study and predict the best MEKC conditions for chiral selectivity using various AABMMs that contain at least one chiral center using various dipeptide combinations of the L-form of alanine, valine and leucine as well as the achiral amino acid glycine. To build this predictive model, experimental data from Nuclear Magnetic Resonance (NMR) studies and MEKC will be combined with the insights gained from the MD simulation studies.

Here, we report an MD simulation study of Dansyl amino acids, including Dansyl-Leucine (Dans-Leu), Dansyl-Norleucine (Dans-Nor), Dansyl-Tryptophan (Dans-Trp) and Dansyl-Phenylalanine (Dans-Phe) binding to poly (SULV) to further the knowledge of a previous study that examined the same aforementioned systems with experimental MEKC and NOESY NMR [22]. The chemical structures of these compounds are shown in Figures 1(b)-(e). The Dansyl amino acid analytes examined in this study can be divided into two main groups: aliphatic (Dans-Leu and Dans-Nor), and aromatic (Dans-Phe and Dans-Trp). This data is important to incorporate into the QSERR model in the future because it expands the depth and diversity of the analyte training set by introducing chiral analytes with varying characteristics; this will ultimately improve the predictions of the QSERR model. Combining experimental MEKC and NOESY NMR data with computational MD simulation studies will allow for the elucidation of these chiral separation mechanisms and helps to further the knowledge base that is necessary to develop a predictive QSERR database.

\section{Experimental Details}

\section{Building the poly (SULV) micelle computationally}

Molecular modeling and MD simulation methods employed in this research 
project have been reported in detail in previous work from our group [5] [17] [18] [19] [22]. The methods for this research project are summarized as follows. First, a monomer unit of $N$-undecanoyl-(L)-Leucylvalinate (ULV) molecule was built with an overall net charge of -1 within the graphical interface, Xleap, within the AMBER18 software package (see Figure 1(a)) [23]. The $N$-undecanoyl and (L)-Leucylvalinate, represent the surfactant's hydrocarbon tail and dipeptide head group, respectively. The ULV monomer unit then serves as the template surfactant for building the polymerized micelle. Previous fluorescence quenching experiments have shown that poly (SULV) contains on average approximately 20 surfactant monomer units [24]. For this reason, molecular modelling of poly (SULV) was made by covalently linking 20 ULV surfactants at their terminal carbon atom on $\mathrm{N}$-undecanoyl in a linear fashion. The linear chain of 20 ULV surfactants units thus yields an overall net charge of -20 . The two terminal surfactant units on either ends of the linear chain, denoted as ULV1 and ULV20, were then covalently linked at their terminal carbon atom on $\mathrm{N}$-undecanoyl to ultimately form a polymer ring, which is representative of the poly (SULV) molecular micelle. Experiments in our group have determined that the polymer ring allows for the proper equilibration of all amino acid-based molecular micelles with the dipeptide combinations of the L-form of alanine, valine and leucine, and achiral glycine, which is necessary to proceed with the long-term study and development of the QSERR model.

After the poly (SULV) molecular micelle was created, AMBER18 was then used to run a 480.0 ns simulation on a system containing the poly (SULV) molecular micelle and 20 sodium counterions, all of which were solvated with TIP3P water molecules within a volume of a 10 Angstrom truncated octahedron. The average structure of poly (SULV) was then calculated using the following procedure.

Step 1. The root mean squared deviation (RMSD) of the $480 \mathrm{~ns}$ MD simulation was determined. The RMSD is used to monitor the coordinate changes of a system over time in comparison to a reference set of coordinates. In this case, the reference structure was the initial frame. The RMSD values were calculated with Equation (1).

$$
\mathrm{RMSD}=\sqrt{\frac{\sum_{i=0}^{n}\left[m_{i} *\left(X_{i}-Y_{i}\right)^{2}\right]}{M}}
$$

The number of atoms in the system, the mass of each atom, the coordinate vector for the target atom, the coordinate vector for the reference atom, and the total mass of the system are denoted as $N, m_{i}, X_{i}, Y_{i}$ and $M$, respectively [25] [26]. The RMSD protocol has previously been used in other studies from our group and is summarized as follows [5] [17] [18] [19] [27]. Throughout the 480.0 ns simulation, the poly (SULV) micelle structure will begin to change over time as it tries to settle into its energetically-favorable structure. The RMSD plot is composed of a $\mathrm{Y}$-axis representing the RMSD value, and an $\mathrm{X}$-axis representing 
the simulation time. The RMSD value will initially increase at a rapid rate at the beginning of the simulation then proceed to equilibrate and level off. The leveled-off region in the RMSD plot denotes which frames in the trajectory file contain representative structures of the equilibrated micelle.

Step 2. After determining which frames were associated with the equilibrated region, the average structure was determined from those frames, ultimately yielding an average, equilibrated structure of poly (SULV). This average structure was then used as the reference structure for the subsequent RMSD analysis on the 480 ns trajectory file.

Step 3. To obtain the final equilibrated poly (SULV) molecular micelle structure that was used for this study, the RMSD was calculated on all frames of the $480 \mathrm{~ns}$ trajectory file with the reference frame being the average theoretical structure obtained from Step 2. From here, the frame containing the lowest RMSD value is the representative poly (SULV) molecular micelle structure. The poly (SULV) molecular micelle structure was then stripped of its aqueous solvent consisting of water and sodium ions. The non-solvated, equilibrated structure of poly (SULV) was then used for the ligand docking analyses.

\section{Ligand Docking}

The Molecular Operating Environment (MOE) software package was used to identify the binding pockets on the representative poly (SULV) and dock ligand enantiomers into each of the identified pockets [28]. The MOE software contains a Site Finder module, which was used to identify the binding pockets of poly (SULV); Site Finder utilizes the alpha sphere method to identify molecular cavities and potential binding sites [19] [29]. Alpha spheres are placed within cavities where four receptor atoms sit at its spherical boundaries [30]. Non-polar regions with poor hydrogen bonding capabilities are represented by white spheres. Regions where hydrogen bonding interactions are likely to occur are represented by red spheres [19]. Therefore, the hydrophobicity and hydrophilicity of a binding pocket can be quantified. Site Finder identified three binding pockets within poly (SULV).

MOE was then used to dock the L- and D-enantiomers of Dans-Leu, Dans-Nor, Dans-Phe and Dans-Trp into each of the three binding pockets of poly (SULV). During the docking analysis process, poly (SULV) was kept static, whereas the Dansyl enantiomers being investigated were dynamic in their movement and poses. The Triangle Matcher method within MOE was used to screen various poses of the enantiomer into the binding pocket. The Triangle Matcher method screens ligand poses by aligning three atoms on the enantiomeric compound with three alpha spheres within the binding pocket [31]. For each pose that is screened, the London dG scoring function is used to calculate the binding free energy value [29]. The poses are then ranked based on their dG values, and the highest scoring enantiomer is used for studies in the MD simulation. The $\mathrm{L}$ - and D-enantiomers of each of the four Dansyl amino acids were docked and scored into the three binding pockets of poly (SULV) resulting in a total of $24 \mathrm{MD}$ si- 
mulations to be conducted and analyzed.

MD simulations were conducted on each enantiomer in all three binding pockets of poly (SULV) using AMBER18. The gaff and ff19SB force fields were employed in these simulations. The gaff force field was used to describe the chemical parameters of the surfactant tail constituent and each of the Dansyl enantiomers using the antechamber package within AMBER18 [32]. The ff19SB force field defines the chemical parameters of amino acids and was employed to provide proper parametrization of the amino acid head group of the surfactant [33]. Each MD simulation of the enantiomer to poly (SULV) complex was solvated with 20 sodium ions and solvated with TIP3P water molecules within a volume of a 10 Angstrom truncated octahedron. The MD simulation is summarized as follows. The first step in the MD simulation is initiated with a 50 ps minimization step. This was followed by a 20 ps warm-up step, which allowed the system time to increase to $300 \mathrm{~K}$. The system was then allowed to equilibrate to a pressure of $1 \mathrm{~atm}$ with a time of 20 ps. The final production run for each MD simulation was conducted for $60 \mathrm{~ns}$. Each simulation was monitored for anomalies with the Visual Molecular Dynamics (VMD) software to ensure that the simulation ran properly. This validation method was used to ensure that the enantiomer being investigated did not leave the pocket during the simulation or do anything else unexpected.

\section{Binding Free Energy Analyses}

The binding free energy values $\left(\mathrm{kJ} \cdot \mathrm{mol}^{-1}\right)$ for each enantiomer: poly (SULV) molecular complex were calculated using post-trajectory analysis. The trajectory files of each MD simulation for each molecular complex were used to calculate the $\Delta \mathrm{G}_{\text {binding }}$ values using the Molecular Mechanics-Generalized Born Solvent Accessibility (MM-GBSA) method [34] [35]. Equation (2) shows that the $\Delta \mathrm{G}_{\text {binding }}$ values are calculated for each of the 24 molecular systems by taking the difference between the sum of the separate free energy values of poly (SULV) and the respective enantiomers, with its difference with the poly (SULV): enantiomer complex [5] [18] [19].

$$
\Delta G_{\text {binding }}=\Delta G_{\text {complex }}-\left(\Delta G_{\text {poly (SULV })}+\Delta G_{\text {enantiomer }}\right)
$$

The binding free energy value of each enantiomer in the three pockets of poly (SULV) were used to calculate the percent occupancy, $P_{p}$, which calculates where a given enantiomer is most likely to occupy based on its $\Delta G_{\text {binding }}$ values in each of the binding pockets. The percent population was calculated with Equation (3) [5].

$$
P_{i}=\frac{\mathrm{e}^{-\frac{G_{i}}{K_{B}} * T}}{\sum_{i=1}^{N} \mathrm{e}^{-\frac{G_{i}}{K_{B}} * T}}
$$

The binding free energy of the enantiomer in the $\mathrm{i}^{\text {th }}$ pocket of poly (SULV), Boltzmann's constant, and Kelvin temperature are represented by $G_{D}, K_{B}, T$, respectively. 


\section{Hydrogen Bond Analyses}

The CPPTRAJ utility in AMBER18 was used to perform hydrogen bond analyses on each of the 24 poly (SULV):enantiomer complexes [25]. Hydrogen bonding interactions occur when a hydrogen atom is covalently linked to heavy atoms, such as fluorine, oxygen or nitrogen, and that same hydrogen also shares intermolecular interactions with other nearby heavy atoms (usually fluorine, oxygen or nitrogen). The heavy atom that the hydrogen atom is covalently linked to is known as the donor atom, whereas the acceptor atom is the nearby atom that the hydrogen atom is having intermolecular interactions with. The CPPTRAJ utility is able to analyze each trajectory file and track the hydrogen bonds that are being broken and formed throughout the $60 \mathrm{~ns}$ simulation. It does so by following two main geometric criteria that are characteristic for hydrogen bonds: 1) the distance between the donor-to-acceptor heavy atoms must be within $3 \AA$ of each other, and 2) the donor-hydrogen-acceptor angle cutoff must be $\pm 30^{\circ}$ [5].

\section{Discussion}

Previously reported data shows that when using poly (SULV) as the pseudostationary phase in MEKC experiments in conjunction with the L- and D-enantiomers of Dans-Leu, Dans-Nor, Dans-Trp and Dans-Phe, the L-enantiomer of each of these compounds eluted second [22]. This indicates that the L-enantiomers of each of the Dansyl amino acids studied have stronger binding interactions with poly (SULV) than the D-enantiomers. The previous study also investigated the interactions between the Dansyl amino acids and poly (SULV) using NOESY NMR. However, due to spectral overlap in the corresponding NOESY NMR spectra between the Dansyl amino acids and poly (SULV), the NOE interactions were not fully determined [22]. By investigating the interactions between the Dansyl amino acids and poly (SULV) via MD simulation studies, molecular-level insight will be able to provide information about the molecular interactions that govern chiral recognition in these molecular systems.

As mentioned previously, the MOE software package identified poly (SULV) as having three binding pockets, which can be found in Figure 2. Additionally, each pocket in relation to each other in poly (SULV) is shown in Figure 3. The binding free energy values and the occupancy percentages for each pocket with each of the Dansyl amino acid enantiomers are summarized in Table 1. The binding free energy data for each Dansyl amino acid will be summarized in the following sections.

Furthermore, previous studies also showed that experimental NOESY NMR data was inconclusive in determining which interactions governed chiral selectivity due to spectral overlap between the NOE intensities of the Dansyl amino acids and poly (SULV) [22]. Therefore, hydrogen bond analyses will be used to rationalize why the L-enantiomers of the Dansyl amino acids bind stronger to poly (SULV) than the D-enantiomers, while providing molecular-level insight 


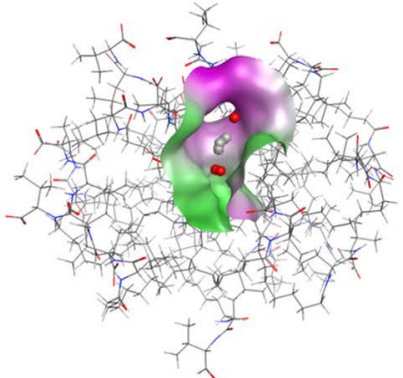

Pocket 1

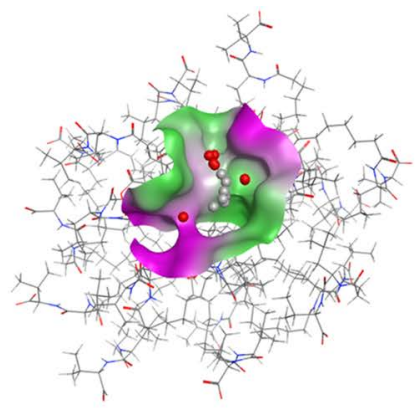

Pocket 2

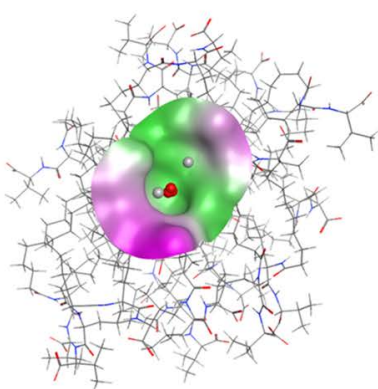

Pocket 3

Figure 2. Poly (SULV) binding pockets identified via MOE analysis. Three binding pockets were identified and populated with alpha centers. The lipophilic and hydrophilic regions of the pocket are denoted by green and purple, respectively.

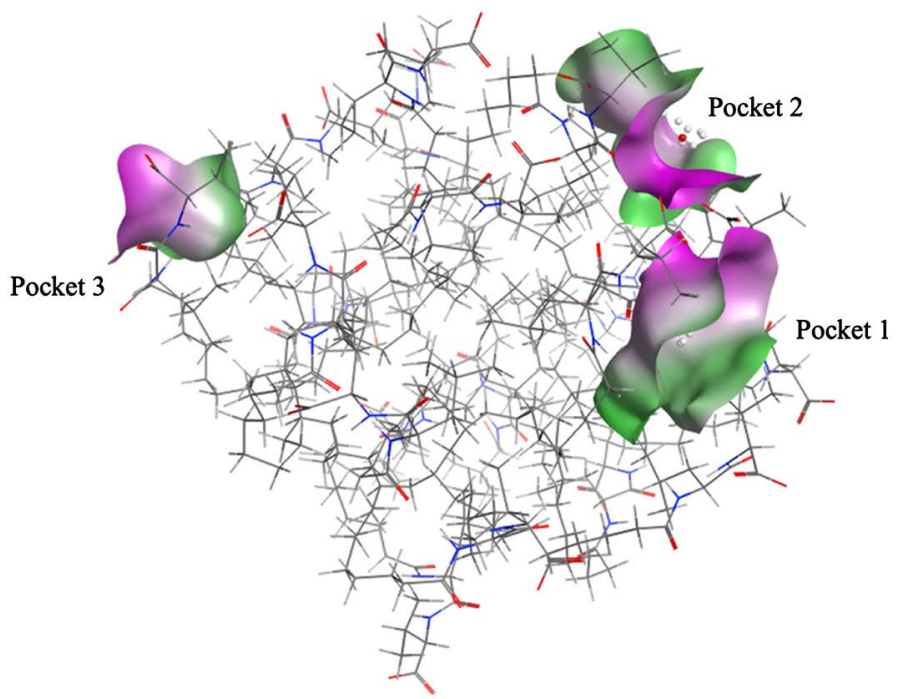

Figure 3. Spatial orientation of the three Poly (SULV) binding pockets in respect to each other.

into the specific bonding interactions that govern chiral selectivity in these systems. The trajectory files for each molecular system will be analyzed for its intermolecular hydrogen bonds that are formed throughout the simulation. The hydrogen bonding analyses will provide detailed information about the acceptor atoms and donor atoms that are participating in hydrogen bonding [5] [18] [19]. Additionally, the highest number of consecutive frames that a hydrogen bond is maintained will be denoted as the 'max lifetime' for that specific hydrogen bond; this provides insight into the frequency of the hydrogen bond being formed [36]. The hydrogen bond occupancy represents the percentage of frames that a hydrogen bond is maintained throughout an entire simulation. Table 2 displays bonds that had greater than a $10 \%$ hydrogen bond occupancy throughout the simulations. All hydrogen bond occupancies less than $10 \%$ were considered negligible and therefore did not contribute greatly to chiral selectivity. In the following sections, the binding free energy values for each Dansyl amino acid will be rationalized using the data collected from hydrogen bonding analyses. 
Table 1. Binding free energy values and pocket percent occupied for each of the Dansyl amino acid enantiomers binding to poly (SULV).

\begin{tabular}{|c|c|c|c|}
\hline & Pocket 1 & Pocket 2 & Pocket 3 \\
\hline \multicolumn{4}{|l|}{ Dansyl $(L)$ Leucine } \\
\hline$\Delta \mathrm{G}_{\text {binding }}\left(\mathrm{kJ} \cdot \mathrm{mol}^{-1}\right)$ & -21.8938 & -8.4391 & -9.4166 \\
\hline Percent occupied & $98.84 \%$ & $0.50 \%$ & $0.66 \%$ \\
\hline \multicolumn{4}{|l|}{ Dansyl (D) Leucine } \\
\hline$\Delta \mathrm{G}_{\text {binding }}\left(\mathrm{kJ} \cdot \mathrm{mol}^{-1}\right)$ & -14.5811 & 0.1686 & -9.3757 \\
\hline Percent occupied & $87.84 \%$ & $1.28 \%$ & $10.89 \%$ \\
\hline \multicolumn{4}{|l|}{ Dansyl (L) Norleucine } \\
\hline$\Delta \mathrm{G}_{\text {binding }}\left(\mathrm{kJ} \cdot \mathrm{mol}^{-1}\right)$ & -22.1763 & -13.6191 & -12.2257 \\
\hline Percent occupied & $95.17 \%$ & $3.07 \%$ & $1.76 \%$ \\
\hline \multicolumn{4}{|l|}{ Dansyl $(D)$ Norleucine } \\
\hline$\Delta \mathrm{G}_{\text {binding }}\left(\mathrm{kJ} \cdot \mathrm{mol}^{-1}\right)$ & -15.9457 & -7.8426 & -14.2665 \\
\hline Percent occupied & $64.57 \%$ & $2.50 \%$ & $32.93 \%$ \\
\hline \multicolumn{4}{|l|}{ Dansyl (L) Tryptophan } \\
\hline$\Delta \mathrm{G}_{\text {binding }}\left(\mathrm{kJ} \cdot \mathrm{mol}^{-1}\right)$ & -18.8091 & -11.5680 & -21.3329 \\
\hline Percent occupied & $26.27 \%$ & $1.44 \%$ & $72.29 \%$ \\
\hline \multicolumn{4}{|c|}{ Dansyl(D) Tryptophan } \\
\hline$\Delta \mathrm{G}_{\text {binding }}\left(\mathrm{kJ} \cdot \mathrm{mol}^{-1}\right)$ & -8.6631 & -13.6408 & -11.7956 \\
\hline Percent occupied & $8.42 \%$ & $62.00 \%$ & $29.58 \%$ \\
\hline \multicolumn{4}{|c|}{ Dansyl (L) Phenylalanine } \\
\hline$\Delta \mathrm{G}_{\text {binding }}\left(\mathrm{kJ} \cdot \mathrm{mol}^{-1}\right)$ & -12.4774 & -10.2752 & -13.3349 \\
\hline Percent occupied & $35.41 \%$ & $14.64 \%$ & $49.95 \%$ \\
\hline \multicolumn{4}{|c|}{ Dansyl (D) Phenylalanine } \\
\hline$\Delta \mathrm{G}_{\text {binding }}\left(\mathrm{kJ} \cdot \mathrm{mol}^{-1}\right)$ & -11.5032 & - & -12.0959 \\
\hline Percent occupied & $44.08 \%$ & $0.00 \%$ & $55.92 \%$ \\
\hline
\end{tabular}

Table 2. Hydrogen bonds formed between Poly (SULV) and aliphatic Dansyl amino acids, consisting of Dans-Leu and Dans-Nor.

\begin{tabular}{|c|c|c|c|}
\hline Acceptor atom & Donor atom & Max Lifetime & $\begin{array}{c}\text { Hydrogen Bond } \\
\text { Occupancy }\end{array}$ \\
\hline \multicolumn{4}{|l|}{ Dansyl- $(L)$-Leucine pocket one } \\
\hline Dans-Leu $\mathrm{C}=\mathrm{O}$ & MM chain 16: Leu NH & 4895 & $46.36 \%$ \\
\hline MM chain 18: Val $\mathrm{C}=\mathrm{O}$ & Dans-Leu NH & 1320 & $17.83 \%$ \\
\hline MM chain 18: Leu $\mathrm{C}=\mathrm{O}$ & Dans-Leu NH & 345 & $10.41 \%$ \\
\hline \multicolumn{4}{|c|}{ Dansyl-(D)-Leucine pocket one } \\
\hline MM chain 17: Leu $C=O$ & Dans-Leu NH & 1131 & $33.68 \%$ \\
\hline Dans-Leu $\mathrm{C}=\mathrm{O}$ & MM chain 20: Leu NH & 1737 & $14.22 \%$ \\
\hline
\end{tabular}




\section{Continued}

Dansyl-(L)-Norleucine pocket one

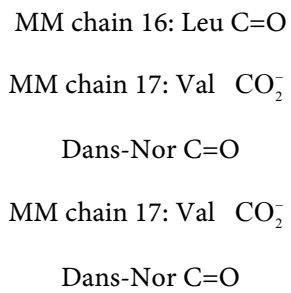

Dansyl-(D)-Norleucine pocket one

$\mathrm{MM}$ chain 17: Val $\mathrm{CO}_{2}^{-}$

$\begin{array}{ccc}\text { Dans-Nor NH } & 2838 & 61.41 \% \\ \text { Dans-Nor OH } & 132 & 45.66 \% \\ \text { MM chain 17: Leu NH } & 4358 & 39.47 \% \\ \text { Dans-Nor OH } & 99 & 25.71 \% \\ \text { MM chain 17: Val NH } & 4026 & 21.26 \% \\ & & \\ & & \end{array}$

\section{Dans-Leu binding to poly (SULV)}

Dansyl-(L)-Leucine and Dansyl-(D)-Leucine were docked into each of the three binding pockets identified in poly (SULV) as shown in Figures 4(a)-(f). Table 1 shows that for Dansyl-(L)-Leucine, the binding free energy values for poly (SULV) pockets one, two and three are: $-21.8938,-8.4391$ and -9.4166 $\mathrm{kJ} \cdot \mathrm{mol}^{-1}$, respectively. The percent occupancies can be calculated by employing Equation 3 to this data set. The percent occupancy for Dansyl-(L)-Leucine in pockets one, two and three of poly (SULV) are: $98.84 \%, 0.50 \%$ and $0.66 \%$, respectively. Based on this data, it is evident that Dansyl-(L)-Leucine clearly favors pocket one as shown by its binding free energy value and percent occupancy compared to the other binding pockets. The data indicates that Dansyl-(L)-Leucine does not favor pockets two and three, as this enantiomer has less than $1 \%$ occupancy in those locations based on its unfavorable binding free energy values, when compared to pocket one. Therefore, pockets two and three can be considered so low they are nearly negligible.

Table 1 indicates that the binding free energy values of Dansyl-(D)-Leucine to pockets one, two and three of poly (SULV) are $-14.5811,0.1686$ and -9.3757 $\mathrm{kJ} \cdot \mathrm{mol}^{-1}$, respectively. The percent occupancy for Dansyl-(D)-Leucine in pockets one, two and three of poly (SULV) are $87.84 \%, 1.28 \%$ and $10.89 \%$, respectively. It is evident that of the three pockets available, Dansyl-(D)-Leucine significantly favors pocket one, whereas this enantiomer does not favor pockets two or three.

Since Dansyl-(L)-Leucine had the highest percent occupancy in pocket one with a binding free energy value of $-21.8938 \mathrm{~kJ} \cdot \mathrm{mol}^{-1}$, this will be compared to the pocket and binding free energy values that Dansyl-(D)-Leucine favored most. In this case, Dansyl-(D)-Leucine also had the highest percent occupancy in pocket one with a binding free energy value of $-14.5811 \mathrm{~kJ} \cdot \mathrm{mol}^{-1}$. When comparing the two binding free energy values, it is evident that Dansyl-(L)-Leucine has a more negative or stronger binding interaction to poly (SULV) when compared to Dansyl-(D)-Leucine. Therefore, analysis of the MD simulations show that Dansyl-(L)-Leucine would elute second, thus matching experimental MEKC data showing that the L-enantiomer of Dans-Leu elutes second when using 
(a)

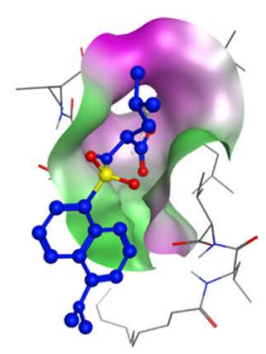

Pocket 1

(d)

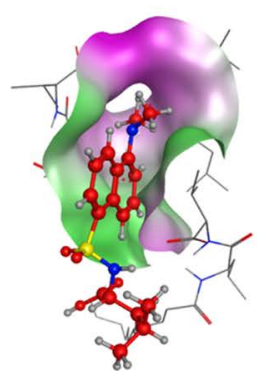

(b)

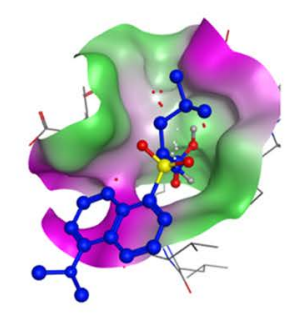

Pocket 2

(e)

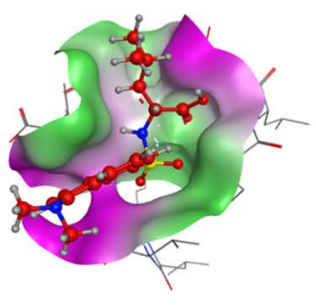

(c)

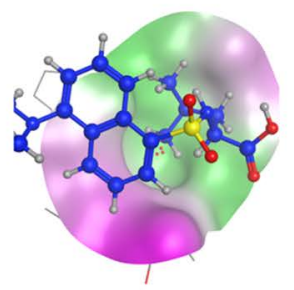

Pocket 3

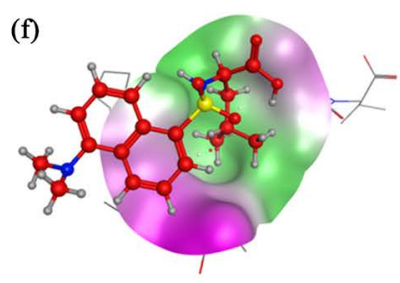

Figure 4. The highest scoring docked position of Dansyl-(L)-Leucine (blue) in each binding pocket of Poly (SULV) is displayed in (a) Pocket 1 (b) Pocket 2 and (c) Pocket 3. The highest scoring docked position of Dansyl-(D)-Leucine (red) in each binding pocket of Poly (SULV) is displayed in (d) Pocket 1 (e) Pocket 2 and (f) Pocket 3.

poly (SULV) as the pseudostationary phase.

As confirmed by the binding free energy values in Table 1, Dansyl-(L)-Leucine binds stronger to poly (SULV) than Dansyl-(D)-Leucine. It is evident that both of the Dans-Leu enantiomers prefer pocket one of poly (SULV) when compared to the remaining two pockets. It is, therefore, important to evaluate and compare the hydrogen bonds that are formed between both enantiomers and poly (SULV) to fully understand why Dansyl-(L)-Leucine binds stronger to poly (SULV) than Dansyl-(D)-Leucine. Table 2 outlines the hydrogen bonding interactions formed between poly (SULV) and both enantiomers of Dans-Leu.

For Dansyl-(L)-Leucine, three major hydrogen bonds were identified. The carbonyl on Dans-Leu acted as the acceptor atom, which interacted with the Leucine N-H of poly (SULV). This bond had the longest max lifetime of 4895 frames, as well as the highest hydrogen bond occupancy of $46.36 \%$. The second most prominent hydrogen bond formed between the Valine carbonyl on poly (SULV) which acted as the acceptor atom for Dans-Leu N-H; this had a max lifetime of 1320 frames, and a hydrogen bond occupancy of $17.83 \%$. The third most prominent hydrogen bond formed between the Leucine carbonyl on poly (SULV) and the Dans-Leu N-H with a max lifetime of 345 frames and a hydrogen bond occupancy of $10.41 \%$. Based on this data, it is evident that the key interaction is the bond formed between the carbonyl on Dans-Leu and the poly (SULV) Leucine N-H. This suggests that Dansyl-(L)-Leucine preferentially binds to the inner amino acid of poly (SULV), thus interacting more within the core of the micelle rather than its exterior amino acid, Valine.

In Dansyl-(D)-Leucine, two major hydrogen bonds were identified through- 
out the simulation. The most prominent hydrogen bond formed between the Leucine carbonyl on poly (SULV) and the Dans-Leu N-H with a max lifetime of 1131 frames and a hydrogen bond occupancy of 33.68\%. The second most prominent hydrogen bond for Dansyl-(D)-Leucine was formed between the carbonyl on Dans-Leu and the poly (SULV) Leu N-H bond with a max lifetime of 1737 frames and a hydrogen bond occupancy of $14.22 \%$. While this bond had a longer max lifetime it was not sustained quite as long throughout the simulation as the other aforementioned hydrogen bond interaction. It is evident that the hydrogen bond formed between the Leucine carbonyl on poly (SULV) and the Dans-Leu N-H contributed the most to the binding free energy value, and similar to Dansyl-(L)-Leucine, it also preferentially binds to the inner amino acid head group of poly (SULV).

When comparing the hydrogen bonding analysis data for Dansyl-(L)-Leucine and Dansyl-(D)-Leucine, it is evident that more hydrogen bonds are able to be formed with the L-enantiomer than the $\mathrm{D}$-enantiomer, and for a longer period of time. More specifically, Dansyl-(L)-Leucine was able to form three strong intermolecular hydrogen bonds with poly (SULV) compared to the two intermolecular hydrogen bonds formed with Dansyl-(D)-Leucine in pocket one of poly (SULV). Therefore, the hydrogen bonding interactions justify the stronger binding free energy values of Dansyl-(L)-Leucine to poly (SULV) than that of Dansyl-(D)-Leucine. For both enantiomers, the preferred binding interactions are near the inner amino acid head group of poly (SULV), suggesting its significance in separating the Dans-Leu enantiomers.

\section{Dans-Nor binding to poly (SULV)}

Dansyl-(L)-Norleucine and Dansyl-(D)-Norleucine were docked into each of the three binding pockets identified in poly (SULV) as shown in Figures 5(a)-(f). Table 1 shows that for Dansyl-(L)-Norleucine, the binding free energy values for poly (SULV) pockets one, two and three are: $-22.1763,-13.6191$ and -12.2257 $\mathrm{kJ} \cdot \mathrm{mol}^{-1}$, respectively. The percent occupancy for Dansyl-(L)-Norleucine in pockets one, two and three of poly (SULV) are: $95.17 \%, 3.07 \%$ and $1.76 \%$, respectively. Based on this data, it is evident that Dansyl-(L)-Norleucine favors pocket 1 as shown by its binding free energy value and percent occupancy compared to the other binding pockets. The data indicates that Dansyl-(L)-Norleucine does not favor binding pockets two and three, as this enantiomer has a range of approximately $1-3 \%$ for occupying those binding pockets based on its unfavorable binding free energy values.

Table 1 also indicates that the binding free energy values of Dansyl-(D)-Norleucine to binding pockets one, two and three of poly (SULV) are $-15.9457,-7.8426$ and $-14.2665 \mathrm{~kJ} \cdot \mathrm{mol}^{-1}$, respectively. The percent occupancy for Dansyl-(D)-Norleucine in pockets one, two and three of poly (SULV) are $64.57 \%, 2.50 \%$ and $32.93 \%$, respectively. It is evident that of the three pockets available, Dansyl-(D)-Norleucine favors pocket one, whereas this enantiomer 
(a)

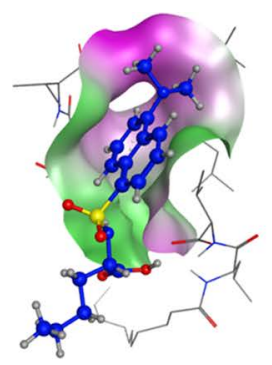

Pocket 1

(d)

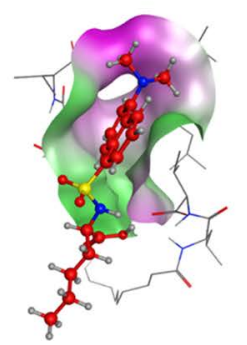

(b)

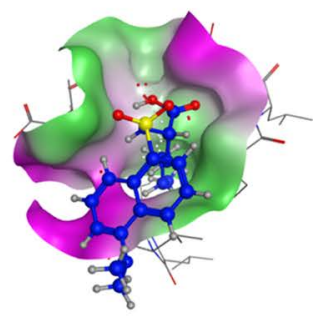

Pocket 2

(e)

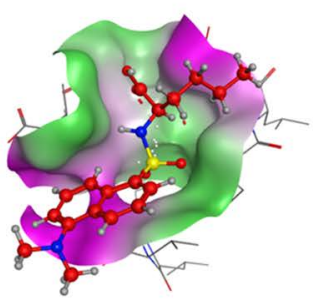

(c)

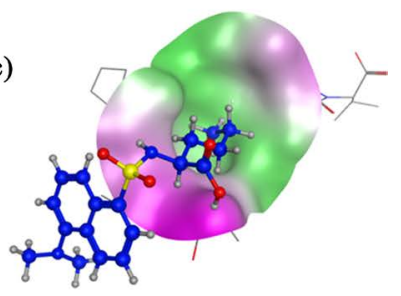

Pocket 3

(f)

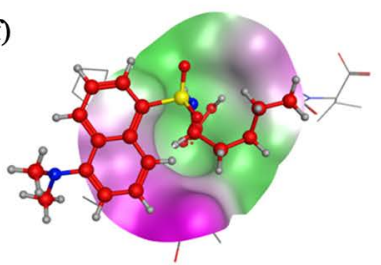

Figure 5. The highest scoring docked position of Dansyl-(L)-Norleucine (blue) in each binding pocket of Poly (SULV) is displayed in (a) Pocket 1 (b) Pocket 2 and (c) Pocket 3. The highest scoring docked position of Dansyl-(D)-Norleucine (red) in each binding pocket of Poly (SULV) is displayed in (d) Pocket 1 (e) Pocket 2 and (f) Pocket 3.

does not favor pocket three. Approximately one-third of the simulation time, Dansyl-(D)-Norleucine might spend time in pocket two, however it significantly favors pocket one.

Since Dansyl-(L)-Norleucine had the highest percent occupancy in pocket one with a binding free energy value of $-22.1763 \mathrm{~kJ} \cdot \mathrm{mol}^{-1}$, this will be compared to the pocket and binding free energy values that Dansyl-(D)-Norleucine favored most. In this case, Dansyl-(D)-Norleucine also had the highest percent occupancy in pocket one with a binding free energy value of $-15.9457 \mathrm{~kJ} \cdot \mathrm{mol}^{-1}$. When comparing the two binding free energy values of both enantiomers in their most favored pockets, it is evident that Dansyl-(L)-Norleucine has a more negative or stronger binding interaction to poly (SULV) as compared to its enantiomer, Dansyl-(D)-Norleucine. Therefore, analysis of the MD simulations show that Dansyl-(L)-Norleucine would elute second, which is consistent with experimental MEKC data when using poly (SULV) as the pseudostationary phase to separate the enantiomers of Dans-Nor.

As confirmed by the binding free energy values in Table 1, Dansyl-(L)-Norleucine binds stronger to poly (SULV) than Dansyl-(D)-Norleucine. It is also evident that both of the Dans-Nor enantiomers prefer pocket one of poly (SULV). It is, therefore, important to evaluate and compare the hydrogen bonds that are formed between both enantiomers and poly (SULV) to fully understand why Dansyl-(L)-Norleucine binds stronger to poly (SULV) than Dansyl-(D)-Norleucine. Table 2 outlines the hydrogen bonding interactions formed between poly (SULV) and both enantiomers of Dans-Nor. The data is summarized as follows. 
The most prominent hydrogen bond in Dansyl-(L)-Norleucine with poly (SULV) pocket one was formed between the carbonyl of Leucine in poly (SULV) with the N-H bond on Dans-Nor. This bond had a max lifetime of 2838 frames and a hydrogen bond occupancy of $61.41 \%$. Dansyl-(L)-Norleucine also had an additional four other hydrogen bonds formed, in which the details on their interactions are outline in Table 2. Thus, Dansyl-(L)-Norleucine had a total of five intermolecular hydrogen bonds within pocket one of poly (SULV), which had preferential binding to the inner amino acid head group of poly (SULV), which is Leucine.

Dansyl-(D)-Norleucine only had one prominent hydrogen bond, which was between the terminal carboxylate group on the outer amino acid, Valine, in poly (SULV) and the O-H group on Dans-Nor. This bond had a max lifetime of 215 with a hydrogen bond occupancy of $33.28 \%$. Since this had a lower binding energy value, it is possible that its conformation did not allow it to penetrate as deeply into the micellar core to interact with the inner amino acid head group.

In conclusion, Dansyl-(L)-Norleucine was able to form significantly more hydrogen bonds and for longer periods of time than that of Dansyl-(D)-Norleucine in pocket one of poly (SULV). More specifically, Dansyl-(L)-Norleucine was able to form a total of five intermolecular hydrogen bonds with poly (SULV), whereas Dansyl-(L)-Norleucine only formed one. This ultimately contributes to the stronger binding free energy value seen in Dansyl-(L)-Norleucine when compared to the D-enantiomer binding to poly (SULV).

\section{Dans-Trp binding to poly (SULV)}

Dansyl-(L)-Tryptophan and Dansyl-(D)-Tryptophan were docked into each of the three binding pockets identified in poly (SULV) as shown in Figures 6(a)-(f). Table 1 shows that for Dansyl-(L)-Tryptophan, the binding free energy values for poly (SULV) pockets one, two and three are: $-18.8091,-11.5680$ and $-21.3329 \mathrm{~kJ} \cdot \mathrm{mol}^{-1}$, respectively. The percent occupancy for Dansyl-(L)-Tryptophan in pockets one, two and three of poly (SULV) are: $26.27 \%, 1.44 \%$ and $72.29 \%$, respectively. Based on this data, it is evident that Dansyl-(L)-Tryptophan favors pocket three as shown by its binding free energy value and percent occupancy compared to the other binding pockets. The data indicates that Dansyl-(L)Tryptophan does not favor binding pocket two as this enantiomer has a less than 2\% occupying for this site. Dansyl-(L)-Tryptophan would spend approximately a quarter of its simulation time in pocket one. On the other hand, most of the time this enantiomer favors pocket three based on its binding percent occupancy.

Table 1 indicates that the binding free energy values of Dansyl-(D)-Tryptophan to binding pockets one, two and three of poly (SULV) are $-8.6631,-13.6408$ and $-11.7956 \mathrm{~kJ} \cdot \mathrm{mol}^{-1}$, respectively. The percent occupancy for Dansyl-(D)-Tryptophan in pockets one, two and three of poly (SULV) are $8.42 \%, 62.00 \%$ and $29.58 \%$, respectively. It is evident that of the three total pockets available, Dansyl-(D)-Tryptophan favors pocket two, whereas this enantiomer does not favor 
(a)

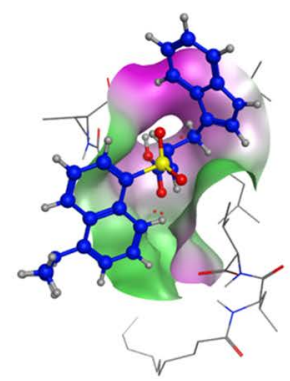

Pocket 1

(d)

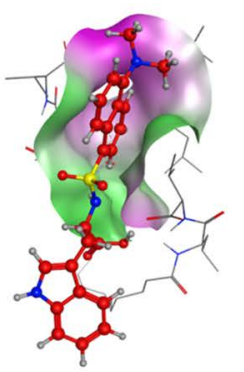

(b)

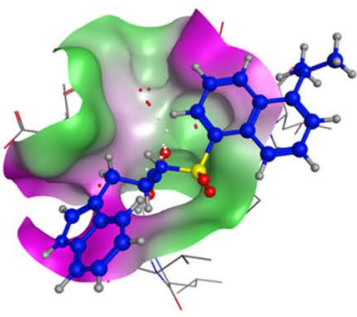

Pocket 2

(e)

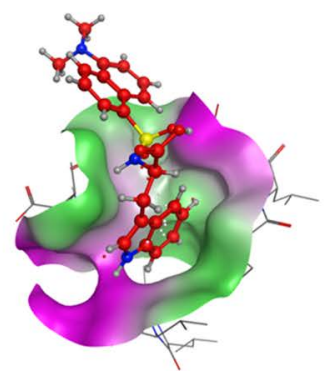

(c)

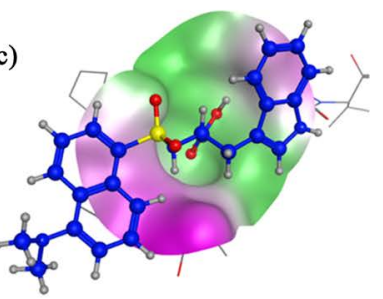

Pocket 3

(f)



Figure 6. The highest scoring docked position of Dansyl-(L)-Tryptophan (blue) in each binding pocket of Poly (SULV) is displayed in (a) Pocket 1 (b) Pocket 2 and (c) Pocket 3. The highest scoring docked position of Dansyl-(D)-Tryptophan (red) in each binding pocket of Poly (SULV) is displayed in (d) Pocket 1 (e) Pocket 2 and (f) Pocket 3.

pocket one and shows some favorability for pocket three.

Dansyl-(L)-Tryptophan had the highest percent occupancy in pocket three with a binding free energy value of $-21.3329 \mathrm{~kJ} \cdot \mathrm{mol}^{-1}$, therefore, this will be compared to the pocket and binding free energy values that Dansyl-(D)-Tryptophan favor most. In this case, Dansyl-(D)-Tryptophan had the highest percent occupancy (62\%) in pocket two with a binding free energy value of $-13.6408 \mathrm{~kJ} \cdot \mathrm{mol}^{-1}$. When comparing the two binding free energy values of both enantiomers in their favored poly (SULV) pockets, it is evident that Dansyl-(L)-Tryptophan has a more negative or stronger binding interaction to poly (SULV) than that of the Dansyl-(D)-Tryptophan enantiomer. This data is consistent with experimental MEKC elution data because when separating Dans-Trp enantiomers using poly (SULV) as the pseudostationary phase, the L-enantiomer elutes second.

As confirmed by the binding free energy values in Table 1, Dansyl-(L)Tryptophan binds stronger to poly (SULV) than Dansyl-(D)-Tryptophan. It is also evident that Dansyl-(L)-Tryptophan prefers pocket three of poly (SULV), whereas Dansyl-(D)-Tryptophan prefers pocket two. It is, therefore, important to evaluate and compare the hydrogen bonds that are formed between both enantiomers in those respective pockets of poly (SULV) to fully understand why Dansyl-(L)-Tryptophan binds stronger to poly (SULV) than Dansyl-(D)-Tryptophan. Table 3 outlines the hydrogen bonding interactions formed between poly (SULV) and both enantiomers of Dans-Trp. The data is summarized as follows.

For Dansyl-(L)-Tryptophan, a total of five intermolecular hydrogen bonds were formed with pocket three of poly (SULV). The most prominent hydrogen 
Table 3. Hydrogen bonds formed between Poly (SULV) and aromatic Dansyl amino acids, consisting of Dans-Trp and Dans-Phe.

\begin{tabular}{|c|c|c|c|}
\hline Acceptor atom & Donor atom & $\begin{array}{c}\text { Max } \\
\text { Lifetime }\end{array}$ & $\begin{array}{l}\text { Hydrogen Bond } \\
\text { Occupancy }\end{array}$ \\
\hline \multicolumn{4}{|c|}{ Dansyl-(L)-Tryptophan pocket three } \\
\hline MM chain 13: $\mathrm{Val} \quad \mathrm{CO}_{2}^{-}$ & Dans-Trp NH & 600 & $61.66 \%$ \\
\hline MM chain 13: $\mathrm{Val} \quad \mathrm{CO}_{2}^{-}$ & Dans-Trp OH & 386 & $61.14 \%$ \\
\hline MM chain 13: $\mathrm{Val} \quad \mathrm{CO}_{2}^{-}$ & Dans-Trp NH & 656 & $35.82 \%$ \\
\hline MM chain 13: $\mathrm{Val} \quad \mathrm{CO}_{2}^{-}$ & Dans-Trp OH & 333 & $35.11 \%$ \\
\hline Dans-Trp OH & MM chain 13: Leu NH & 1955 & $18.76 \%$ \\
\hline \multicolumn{4}{|c|}{ Dansyl-(D)-Tryptophan pocket two } \\
\hline Dans-Trp C $=\mathrm{O}$ & MM chain 6: Leu NH & 4704 & $58.90 \%$ \\
\hline $\mathrm{MM}$ chain $4: \mathrm{Val} \quad \mathrm{CO}_{2}^{-}$ & Dans-Trp OH & 179 & $15.45 \%$ \\
\hline Dans-Trp $\mathrm{C}=\mathrm{O}$ & MM chain 6: Leu NH & 971 & $10.99 \%$ \\
\hline \multicolumn{4}{|c|}{ Dansyl-(L)-Phenylalanine pocket three } \\
\hline MM chain 13: Leu $\mathrm{C}=\mathrm{O}$ & Dans-Phe OH & 9903 & $33.01 \%$ \\
\hline \multicolumn{4}{|c|}{ Dansyl-(D)-Phenylalanine pocket three } \\
\hline $\mathrm{MM}$ chain $12: \mathrm{Val} \quad \mathrm{CO}_{2}^{-}$ & Dans-Phe NH & 1148 & $\sim 3.83 \%$ \\
\hline MM chain 14: $\mathrm{Val} \quad \mathrm{CO}_{2}^{-}$ & Dans-Phe OH & 594 & $\sim 1.98 \%$ \\
\hline $\mathrm{MM}$ chain 14: $\mathrm{Val} \quad \mathrm{CO}_{2}^{-}$ & Dans-Phe NH & 583 & $\sim 1.94 \%$ \\
\hline
\end{tabular}

bonding interactions occurred between the terminal carboxylate group on the outer amino acid, Valine, in poly (SULV) and the N-H bond on Dans-Trp. This hydrogen bond had a max lifetime of 600 frames and a hydrogen bond occupancy of $61.66 \%$. Another strong hydrogen bond worth mentioning is the one formed between the terminal carboxylate group on the outer amino acid, Valine, in poly (SULV) and the O-H moiety on Dans-Trp. This bond had a max lifetime of 386 frames, with a hydrogen bond occupancy of $61.14 \%$

In contrast, Dansyl-(D)-Tryptophan formed 3 intermolecular hydrogens. The most prominent was the bond formed between the carbonyl on Dans-Trp and the Leu N-H group on poly (SULV). This bond had a max lifetime of 4704 frames and a hydrogen bond occupancy of $58.90 \%$.

Therefore, Dansyl-(L)-Tryptophan was able to form significantly more hydrogen bonds and for longer periods of time in pocket three than that of Dansyl-(D)-Tryptophan in pocket two of poly (SULV). Dansyl-(L)-Tryptophan was able to form a total of five intermolecular hydrogen bonds (four of which had bond occupancies of greater then 30\%) with poly (SULV), whereas Dansyl-(D)-Tryptophan only formed three with only one of those higher a higher occupancy than $30 \%$. This ultimately contributes to the stronger binding free energy value seen in Dansyl-(L)-Tryptophan when compared its D-enantiomer 
binding to poly (SULV).

\section{Dans-Phe binding to poly (SULV)}

Dansyl-(L)-Phenylalanine and Dansyl-(D)-Phenylalanine were docked into each of the three binding pockets identified in poly (SULV) as shown in Figures 7(a)-(f). Table 1 shows that for Dansyl-(L)-Phenylalanine, the binding free energy values for poly (SULV) pockets one, two and three are: -12.4774 , -10.2752 and $-13.3349 \mathrm{~kJ} \cdot \mathrm{mol}^{-1}$, respectively. The percent occupancy for Dansyl-(L)-Phenylalanine in pockets one, two and three of poly (SULV) are: $35.41 \%$, $14.64 \%$ and $49.95 \%$, respectively. Based on this data, it is evident that Dansyl-(L)-Phenylalanine favors pocket three, followed closely by pocket one as shown by those binding free energy values and percent occupancies as compared to pocket two. Dansyl-(L)-Phenylalanine ultimately favors pocket three.

Table 1 indicates that the binding free energy values of Dansyl-(D)-Phenylalanine to binding pockets one and three of poly (SULV) are -11.5032 and -12.0959 $\mathrm{kJ} \cdot \mathrm{mol}^{-1}$, respectively. Pocket two was considered unfavorable and thus negligible as the top scoring docked analyte continuously left the pocket when visualizing the trajectory files for those MD simulations. In that case, the second best scored analyte was then tested and also continuously left the binding pocket during simulations. The simulations were viewed using the VMD software. The percent occupancy for Dansyl-(D)-Phenylalanine in pockets one, two and three of poly (SULV) are $44.08 \%, 0.00 \%$ and $55.92 \%$, respectively. It is evident that of the three total pockets available, Dansyl-(D)-Phenylalanine favors pocket three based on its \% occupancy and binding free energy value.

Dansyl-(L)-Phenylalanine had the highest percent occupancy in pocket three

(a)

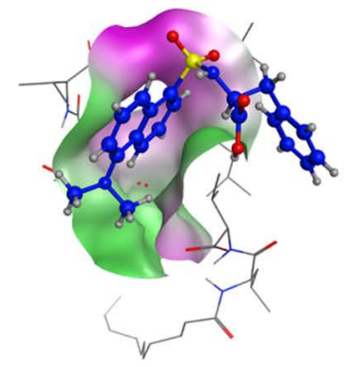

Pocket 1

(d)

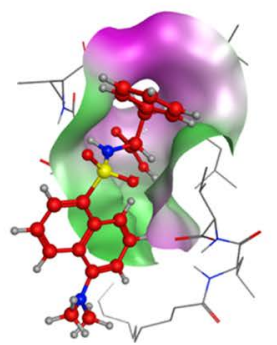

(b)

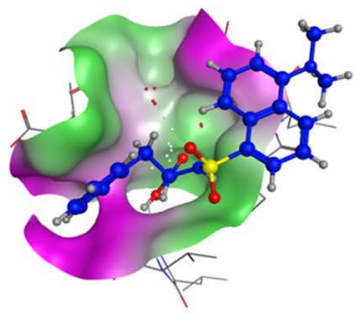

Pocket 2

(e)

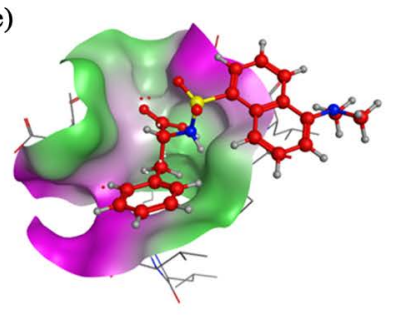

(c)

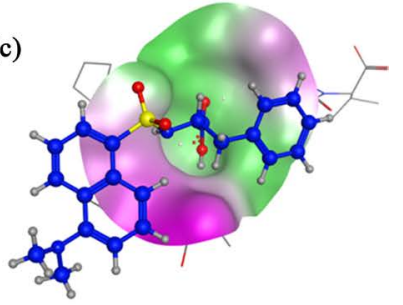

Pocket 3

(f)

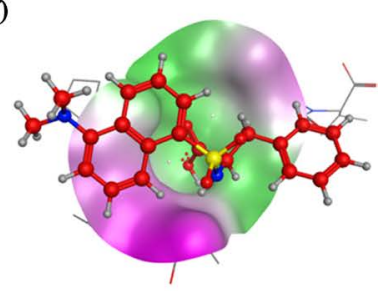

Figure 7. The highest scoring docked position of Dansyl-(L)-Phenylalanine (blue) in each binding pocket of Poly (SULV) is displayed in (a) Pocket 1 (b) Pocket 2 and (c) Pocket 3. The highest scoring docked position of Dansyl-(D)-Phenylalanine (red) in each binding pocket of Poly (SULV) is displayed in (d) Pocket 1 (e) Pocket 2 and (f) Pocket 3. 
with a binding free energy value of $-13.3349 \mathrm{~kJ} \cdot \mathrm{mol}^{-1}$, therefore, this will be compared to the pocket and binding free energy values that Dansyl-(D)-Phenylalanine favor most. In this case, Dansyl-(D)-Phenylalanine also had its highest percent occupancy in pocket three with a binding free energy value of $-12.0959 \mathrm{~kJ} \cdot \mathrm{mol}^{-1}$. When comparing the two binding free energy values of both enantiomers in their favored poly (SULV) pockets, it is evident that Dansyl-(L)-Phenylalanine has a more negative or stronger binding interaction to poly (SULV) than that of the Dansyl-(D)-Phenylalanine enantiomer. Therefore, the L-enantiomer of Dans-Phe would elute second in MEKC studies based on the computational binding free energy values. This data is also consistent with experimental MEKC data because when separating Dans-Phe enantiomers using poly (SULV) as the pseudostationary phase, the L-enantiomer elutes second.

As confirmed by the binding free energy values in Table 1, Dansyl-(L)Phenylalanine binds stronger to poly (SULV) than Dansyl-(D)-Phenylalanine. It is also evident that both enantiomers of Dansyl-Phe prefer pocket three of poly (SULV). It is, therefore, important to evaluate and compare the hydrogen bonds that are formed between both enantiomers in pocket three of poly (SULV) to fully understand why Dansyl-(L)-Phenylalanine binds stronger to poly (SULV) than Dansyl-(D)-Phenylalanine. Table 3 outlines the hydrogen bonding interactions formed between poly (SULV) and both enantiomers of Dans-Phe. The data is summarized as follows.

One major hydrogen bond is notable between Dansyl-(L)-Phenylalanine and poly (SULV), which is formed between the Leu carbonyl on poly (SULV) and the O-H moiety on Dans-Phe. This bond has a max lifetime of 9903 frames and a hydrogen bond occupancy of $33.01 \%$. In contrast, Dansyl-(D)-Phenylalanine did not have any significant hydrogen bonds as all of its hydrogen bond occupancies were below $4.00 \%$.

Therefore, Dansyl-(L)-Phenylalanine was able to form significantly more hydrogen bonds and for longer periods of time in pocket three of poly (SULV) than that of Dansyl-(D)-Phenylalanine. Dansyl-(L)-Tryptophan was able to form one intermolecular hydrogen bond with a relatively long max lifetime, whereas the D-enantiomer did not form any significant hydrogen bonding interactions with pocket three of poly (SULV). This ultimately contributes to the stronger binding free energy value observed in Dansyl-(L)-Phenylalanine when compared its D-enantiomer binding to poly (SULV).

\section{Conclusion}

The binding free energy values for the Dansyl amino acids examined in this study to poly (SULV) were all in agreement with the enantiomeric order determined by MEKC, in which the L-enantiomer of each Dansyl amino acid interacted stronger with poly (SULV) than the D-enantiomer. Spectral overlap in NOESY NMR spectra ultimately led to inconclusive results on the exact interactions that occurred between the Dansyl amino acids binding to poly (SULV). 
Therefore, MD simulations were able to provide molecular-level insight into the hydrogen bonding interactions that govern the chiral separation in these systems. In conclusion, the binding free energy and hydrogen bond analyses studies on poly (SULV) in conjunction with the enantiomers of Dans-Leu, Dans-Nor, Dans-Trp and Dans-Phe were able to provide insight into the molecular interactions that govern chiral selectivity. Furthermore, the computational hydrogen bond analyses supported the computational binding free energy values, which helped to rationalize the reasoning for why the L-enantiomers of each Dansyl amino acid had stronger binding interactions with the amino acid-based molecular micelle, poly (SULV), as opposed to its D-enantiomer. In conclusion, this computation investigation was in agreement with experimental MEKC enantiomer order data, and able to provide insight into the chiral separation mechanisms of these systems. This study also shows that when experimental NMR studies provide inconclusive results of binding interactions due to spectral overlap, MD simulations may be able to supplement information about the underlying chemical interactions.

\section{Acknowledgements}

This work was supported by the following NSF grants under grant numbers: 1708959, 1709394, and 1709680. The research was also supported by Office of Naval Research grants (N00014-18-1-2145 and N00014-19-1-2602), National Institute of Health grant (U54MD007597), and a Robert A. Welch Chemistry Departmental Grant awarded to Texas A\&M University Corpus Christi Chemistry Program. In addition, Dr. Kevin Morris acknowledges the generosity of the Ralph E. Klingenmeyer family.

\section{Conflicts of Interest}

The authors declare no conflicts of interest regarding the publication of this paper.

\section{References}

[1] Nguyen, L.A., He, H. and Pham-Huy, C. (2006) Chiral Drugs: An Overview. International Journal of Biomedical Science, 2, 85-100.

[2] Ananthi, N. (2018) Role of Chirality in Drugs. Organic \& Medicinal Chemistry International Journal, 5, 1. https://doi.org/10.19080/OMCIJ.2018.05.555661

[3] Stolarska, M., Bocian, W., Sitkowski, J., Naumczuk, B., Bednarek, E., Popławska, M., Błażewicz, A. and Kozerski, L. (2020) Cathinones-Routine NMR Methodology for Enantiomer Discrimination and Their Absolute Stereochemistry Assignment, Using R-BINOL. Journal of Molecular Structure, 1219, Article ID: 128575. https://doi.org/10.1016/j.molstruc.2020.128575

[4] Chhabra, N., Aseri, M. and Padmanabhan, D. (2013) A Review of Drug Isomerism and Its Significance. International Journal of Applied and Basic Medical Research, 3, 16-18. https://doi.org/10.4103/2229-516X.112233

[5] Morris, K.F., Billiot, E.J., Billiot, F.H., Ingle, J.A., Krause, K.B., Lewis, C.R., Lipko- 
witz, K.B., Southerland, W.M. and Fang, Y. (2019) Using Molecular Dynamics Simulations to Identify the Key Factors Responsible for Chiral Recognition by an Amino Acid-Based Molecular Micelle. Journal of Dispersion Science and Technology, 40, 716-727. https://doi.org/10.1080/01932691.2018.1479267

[6] Can Başer, K.H. and Özek, T. (2012) Chapter 22 Analysis of Essential Oils and Fragrances by Gas Chromatography. In: Poole, C.F., Ed., Gas Chromatography, Elsevier, Amsterdam, 519-527. https://doi.org/10.1016/B978-0-12-385540-4.00022-5

[7] Yu, R.B. and Quirino, J.P. (2019) Chiral Selectors in Capillary Electrophoresis: Trends during 2017-2018. Molecules, 24, 1135. https://doi.org/10.3390/molecules24061135

[8] Ramos, Z., Rothbauer, G.A., Turner, J., Lewis, C., Morris, K., Billiot, E., Billiot, F. and Fang, Y. (2019) Comparison of Chiral Recognition of Binaphthyl Derivatives with 1-Undecyl-Leucine Surfactants in the Presence of Arginine and Sodium Counterions. Journal of Chromatographic Science, 57, 54-62.

https://doi.org/10.1093/chromsci/bmy080

[9] Engelhardt, H. and Cuñat-Walter, M.A. (1995) Use of Plate Numbers Achieved in Capillary Electrophoretic Protein Separations for Characterization of Capillary Coatings. Journal of Chromatography $A, 717,15-23$.

https://doi.org/10.1016/0021-9673(95)00485-7

[10] Billiot, E., Macossay, J., Thibodeaux, S., Shamsi, S.A. and Warner, I.M. (1998) Chiral Separations Using Dipeptide Polymerized Surfactants: Effect of Amino Acid Order. Analytical Chemistry, 70, 1375-1381. https://doi.org/10.1021/ac9709561

[11] Malviya, R., Bansal, V., Pal, O. and Sharma, P. (2010) High Performance Liquid Chromatography: A Short Review. Journal of Global Pharma Technology, 2, 22-26.

[12] Escuder-Gilabert, L., Martín-Biosca, Y., Medina-Hernández, M.J. and Sagrado, S. (2014) Cyclodextrins in Capillary Electrophoresis: Recent Developments and New Trends. Journal of Chromatography A, 1357, 2-23. https://doi.org/10.1016/j.chroma.2014.05.074

[13] Mantovani, V., Galeotti, F., Maccari, F. and Volpi, N. (2018) Recent Advances in Capillary Electrophoresis Separation of Monosaccharides, Oligosaccharides, and Polysaccharides. Electrophoresis, 39, 179-189. https://doi.org/10.1002/elps.201700290

[14] Shamsi, S.A., Valle, B.C., Billiot, F. and Warner, I.M. (2003) Polysodium N-Undecanoyl1-leucylvalinate: A Versatile Chiral Selector for Micellar Electrokinetic Chromatography. Analytical Chemistry, 75, 379-387. https://doi.org/10.1021/ac020386r

[15] Billiot, F.H., Billiot, E.J. and Warner, I.M. (2001) Comparison of Monomeric and Polymeric Amino Acid Based Surfactants for Chiral Separations. Journal of Chromatography $A$, 922, 329-338. https://doi.org/10.1016/S0021-9673(01)00865-2

[16] Bordes, R. and Holmberg, K. (2015) Amino Acid-Based Surfactants-Do They Deserve More Attention? Advances in Colloid and Interface Science, 222, 79-91. https://doi.org/10.1016/j.cis.2014.10.013

[17] Billiot, A., Fang, Y. and Morris, K. (2019) Characterization of Amino Acid Based Molecular Micelles with Molecular Modeling. Open Journal of Physical Chemistry, 9, 221-240. https://doi.org/10.4236/ojpc.2019.94014

[18] Morris, K.F., Billiot, E.J., Billiot, F.H., Gladis, A.A., Lipkowitz, K.B., Southerland, W.M. and Fang, Y. (2014) A Molecular Dynamics Simulation Study of the Association of 1,1'-Binaphthyl-2,2'-diyl Hydrogenphosphate Enantiomers with a Chiral Molecular Micelle. Chemical Physics, 439, 36-43.

https://doi.org/10.1016/j.chemphys.2014.05.004 
[19] Morris, K.F., Billiot, E.J., Billiot, F.H., Hoffman, C.B., Gladis, A.A., Lipkowitz, K.B., Southerland, W.M. and Fang, Y. (2015) Molecular Dynamics Simulation and NMR Investigation of the Association of the $\beta$-Blockers Atenolol and Propranolol with a Chiral Molecular Micelle. Chemical Physics, 457, 133-146.

https://doi.org/10.1016/j.chemphys.2015.05.024

[20] Lipkowitz, K.B. (2006) Chapter 5 Analysis of Chiral Chromatographic Separations by Molecular Modeling. In: Busch, K.W. and Busch, M.A., Eds., Chiral Analysis, Elsevier, Amsterdam, 97-129. https://doi.org/10.1016/B978-044451669-5/50005-3

[21] Montanari, C.A., Cass, Q.B., Tiritan, M.E. and Souza, A.L.S. (2000) A QSERR Study on Enantioselective Separation of Enantiomeric Sulphoxides. Analytica Chimica Acta, 419, 93-100. https://doi.org/10.1016/S0003-2670(00)00962-4

[22] Valle, B.C., Morris, K.F., Fletcher, K.A., Fernand, V., Sword, D.M., Eldridge, S., Larive, C.K. and Warner, I.M. (2007) Understanding Chiral Molecular Micellar Separations Using Steady-State Fluorescence Anisotropy, Capillary Electrophoresis, and NMR. Langmuir, 23, 425-435. https://doi.org/10.1021/la0612623

[23] Case, D.A., Cheatham, T.E., Darden, T., Gohlke, H., Luo, R., Merz, K.M., Onufriev, A., Simmerling, C., Wang, B. and Woods, R.J. (2005) The Amber Biomolecular Simulation Programs. Journal of Computational Chemistry, 26, 1668-1688. https://doi.org/10.1002/jcc.20290

[24] Billiot, F.H., McCarroll, M., Billiot, E.J., Rugutt, J.K., Morris, K. and Warner, I.M. (2002) Comparison of the Aggregation Behavior of 15 Polymeric and Monomeric Dipeptide Surfactants in Aqueous Solution. Langmuir, 18, 2993-2997. https://doi.org/10.1021/la0110592

[25] Roe, D.R. and Cheatham, T.E. (2013) PTRAJ and CPPTRAJ: Software for Processing and Analysis of Molecular Dynamics Trajectory Data. Journal of Chemical Theory and Computation, 9, 3084-3095. https://doi.org/10.1021/ct400341p

[26] Maiorov, V.N. and Crippen, G.M. (1994) Significance of Root-Mean-Square Deviation in Comparing Three-Dimensional Structures of Globular Proteins. Journal of Molecular Biology, 235, 625-634. https://doi.org/10.1006/jmbi.1994.1017

[27] Morris, K.F., Billiot, E.J., Billiot, F.H., Lipkowitz, K.B., Southerland, W.M. and Fang, Y. (2013) A Molecular Dynamics Simulation Study of Two Dipeptide Based Molecular Micelles: Effect of Amino Acid Order. Open Journal of Physical Chemistry, 3, 20-29. https://doi.org/10.4236/ojpc.2013.31004

[28] Vilar, S., Cozza, G. and Moro, S. (2008) Medicinal Chemistry and the Molecular Operating Environment (MOE): Application of QSAR and Molecular Docking to Drug Discovery. Current Topics in Medicinal Chemistry, 8, 1555-1572. https://doi.org/10.2174/156802608786786624

[29] Galli, C.L., Sensi, C., Fumagalli, A., Parravicini, C., Marinovich, M. and Eberini, I. (2014) A Computational Approach to Evaluate the Androgenic Affinity of Iprodione, Procymidone, Vinclozolin and Their Metabolites. PLOS ONE, 9, e104822. https://doi.org/10.1371/journal.pone.0104822

[30] Le Guilloux, V., Schmidtke, P. and Tuffery, P. (2009) Fpocket: An Open Source Platform for Ligand Pocket Detection. BMC Bioinformatics, 10, 168.

https://doi.org/10.1186/1471-2105-10-168

[31] Cuzzolin, A., Sturlese, M., Malvacio, I., Ciancetta, A. and Moro, S. (2015) DockBench: An Integrated Informatic Platform Bridging the Gap between the Robust Validation of Docking Protocols and Virtual Screening Simulations. Molecules, 20, 9977-9993. https://doi.org/10.3390/molecules20069977

[32] Wang, J., Wolf, R.M., Caldwell, J.W., Kollman, P.A. and Case, D.A. (2004) Devel- 
opment and Testing of a General Amber Force Field. Journal of Computational Chemistry, 25, 1157-1174. https://doi.org/10.1002/jcc.20035

[33] Tian, C., Kasavajhala, K., Belfon, K.A.A., Raguette, L., Huang, H., Migues, A.N., Bickel, J., Wang, Y., Pincay, J., Wu, Q. and Simmerling, C. (2020) ff19SB: Amino-Acid-Specific Protein Backbone Parameters Trained against Quantum Mechanics Energy Surfaces in Solution. Journal of Chemical Theory and Computation, 16, 528-552. https://doi.org/10.1021/acs.jctc.9b00591

[34] Wang, E., Sun, H., Wang, J., Wang, Z., Liu, H., Zhang, J.Z.H. and Hou, T. (2019) End-Point Binding Free Energy Calculation with MM/PBSA and MM/GBSA: Strategies and Applications in Drug Design. Chemical Reviews, 119, 9478-9508. https://doi.org/10.1021/acs.chemrev.9b00055

[35] Ylilauri, M. and Pentikäinen, O.T. (2013) MMGBSA as a Tool to Understand the Binding Affinities of Filamin-Peptide Interactions. Journal of Chemical Information and Modeling, 53, 2626-2633. https://doi.org/10.1021/ci4002475

[36] Chanda, J. and Bandyopadhyay, S. (2006) Hydrogen Bond Lifetime Dynamics at the Interface of a Surfactant Monolayer. The Journal of Physical Chemistry B, 110, 23443-23449. https://doi.org/10.1021/jp065203+ 